\title{
Development of second-generation sample enrichment probe for improved sorptive analysis of volatile organic compounds
}

\author{
B.V. Burger*, M. le Roux, B. Marx, S.A. Herbert, K.T. Amakali \\ Laboratory for Ecological Chemistry, Department of Chemistry and Polymer Science, Stellenbosch University, Private Bag X1, \\ Matieland 7602, South Africa
}

\begin{abstract}
The sample enrichment probe (SEP) has recently been introduced as a user-friendly and cost-effective method for the sorptive extraction of volatile organic analytes from gaseous and aqueous samples for GC and GC-MS analyses. In a further development of the SEP technique, thinner polydimethylsiloxane (PDMS) tubing on polyimide-coated fused silica, instead of stainless steel rods or stalks, were used to produce the second-generation SEPs. The new SEP does not require widening of the needle-guiding orifice of the septum cap and analytes are desorbed at a faster rate from the thinner sleeve, which reduces the risk of carry-over. The flowless period that was previously recommended for analyses of highly volatile analytes is made redundant by the faster desorption from the thinner sorptive medium. It was found that differences in the thermal histories of SEPs are not the cause of the high relative standard deviations (RSDs) reported in our first paper on the technique. Excellent reproducibility can be attained by careful handling and storing of loaded SEPs and by rigorously following a standardised analytical protocol.
\end{abstract}

Keywords: Gas chromatography (GC); Sorptive sampling; Polydimethylsiloxane (PDMS); Sample enrichment probe (SEP); Headspace analysis

\section{Introduction}

Several sorptive sampling methods using polydimethylsiloxane (PDMS) elastomer, commonly known and sold as silicone rubber, have been used for the analysis of volatile organic compounds (VOCs) in various matrices. This polymer is a popular choice of sorbent, owing to its ability to retain apolar and, to an acceptable extent, also polar organic analytes, as well as its thermal stability and the fact that only a limited number of well-characterised PDMS decomposition products are formed during thermal desorption of the trapped volatiles. The first use of PDMS as an absorbent in open tubular traps (OTTs) for the analysis of VOCs was reported by Burger and Munro [1], who utilised a thick lining of PDMS in a capillary column to trap organic compounds from a gaseous sample. Solid-phase microextraction (SPME), developed by Arthur and Pawliszyn [2], is a static sorptive sampling technique in which a thin fibre coated with PDMS is used. This technique has gained general acceptance by analysts, but unfortunately it has a relatively low sensitivity because of the small volume of polymer that is used as sorptive medium.

The sensitivity problem can be circumvented using gum-phase extraction (GPE) in which volatiles are trapped in traps packed with PDMS particles [3]. However, stir bar sorptive extraction (SBSE), developed by Baltussen et al. [4], offers a more elegant solution to the sensitivity problem. In essence, this technique consists of the enrichment of the analytes from an aqueous solution in a sleeve of PDMS rubber on a glass-encapsulated magnetic stir bar. Although the technique was originally developed for the enrichment of analytes from aqueous media it can equally well be used for headspace analyses. Relatively expensive automated thermal desorption and cryotrapping equipment is required to ensure that the desorbed analytes are introduced into the gas chromatographic column as a sharply focussed band. In 2006 a simple technique described as a high-capacity sample enrichment probe (SEP) was introduced for the enrichment of analytes from air and headspace samples [5].

In the SEP technique a relatively large volume of PDMS rubber is also used and in principle this technique is capable of producing results similar to those obtained by SBSE. In this technique the analytes are desorbed in the injector of the gas chromatograph (GC) and cryotrapping is circumvented. Since its introduction this technique has produced excellent qualitative results in many applications in our laboratory. In our opinion the value of the SEP technique lies in its simplicity and the extremely low cost at which qualitative applications, in particular, can be carried out.

Unknown to us PDMS bars were developed specifically for headspace sorptive extraction (HSSE) by Bicchi et al. [6] and Tienpont et al. [7] already in 2000. In some respects HSSE is quite similar to the SEP technique. Earlier attempts at using the SEP in quantitative work produced disappointingly low reproducibilities and it was hypothesised that the unsatisfactory quantitative results that were obtained in multiplicate analyses, using simultaneous enrichment with 
a plurality of SEPS, could probably be ascribed to the different thermal histories of the SEPs that were used [5]. In this communication we report on the further improvement and simplification of the SEP technique and on the development of protocols with which reproducible quantitative results can be obtained.

\section{Experimental}

\section{Instrumentation}

Analyses were carried out on Carlo Erba HRGC and HRGC 5300 GCs (Milan, Italy) and on a Thermo Electron Corporation Trace 2DGC (Milan, Italy) instrument fitted with split/splitless injectors and flame ionisation detectors operated at $230^{\circ} \mathrm{C}$ and $280^{\circ} \mathrm{C}$, respectively. The injectors were used in the split mode. Standard 4-mm i.d. and 5$\mathrm{mm}$ i.d. glass injector liners were used in the Carlo Erba and Trace instruments, respectively. The Trace GC was operated in the single-dimensional mode. A fused silica column (30 m × $0.25 \mathrm{~mm}$ i.d.) coated with PS-255 (DB-1 equivalent) at a film thickness of $1.2 \mu \mathrm{m}$, and made with a 5-m retention gap, was used for experiments on these instruments. The columns were programmed from $30{ }^{\circ} \mathrm{C}$ to $280^{\circ} \mathrm{C}$ at $4{ }^{\circ} \mathrm{C} / \mathrm{min}$. Hydrogen was used as carrier gas at a linear flow velocity of $50 \mathrm{~cm} / \mathrm{s}$ at $40{ }^{\circ} \mathrm{C}$ and the GCs were operated under constant pressure conditions. The data output of the Carlo Erba GCs was processed with Jasco-Borwin software, version 1.5 (Easton, MD), and Chrom-Card software, version 2.4.0, was used to process data on the Trace instrument

\section{Sample enrichment probes}

Two SEP designs were used in the present investigation: Type 1, having fused silica stalks and Type 2 with shorter paramagnetic stainless steel stalks (Fig. 1). Silastic laboratory tubing (PDMS tubing) $(0.64 \mathrm{~mm}$ i.d. $\times 1.19 \mathrm{~mm}$ o.d.) (Dow Corning, Midland, MI) was cut into lengths of exactly $30 \mathrm{~mm}$ (mass ca. $0.028 \mathrm{~g}$ ). These sections of tubing (PDMS sleeves) were weighed to five decimal places, and groups having a mass variance of less than $0.14 \%$ were selected to manufacture SEP30s, i.e. SEPs with sleeves $30 \mathrm{~mm}$ in length. Fused silica tubing (0.7 mm o.d.) (Polymicro, Phoenix, AZ) was cut into lengths of about $130 \mathrm{~mm}$ and sealed off at both ends with an oxy-propane burner to produce stalks for Type 1 SEPs. Using ethanol as lubricating agent, each of these PDMS sleeves was gently slipped over the tip of a fused silica stalk and positioned with one end of the sleeve about $1 \mathrm{~mm}$ from the tip of the stalk.

Care was taken not to stretch or compress the sleeves on their stalks. Type 2 SEP30s with shorter paramagnetic stainless steel stalks $(100 \mathrm{~mm} \times 0.66 \mathrm{~mm})$ instead of fused silica stalks were made from guitar string (PL026, D'Addario, Farmingdale, NY). The Types 2 SEPs were made with sleeves positioned either $1 \mathrm{~mm}$ or $20 \mathrm{~mm}$ from the tips of the stalks. The completed SEPs were placed in a GC oven at $60^{\circ} \mathrm{C}$ for $2 \mathrm{~h}$ to remove any residual ethanol and then conditioned overnight in a GC injector under hydrogen flow at $230{ }^{\circ} \mathrm{C}$ before use. A spherical NdFeB magnet $(3 \mathrm{~mm})$ glued to a stainless steel tube $(2 \mathrm{~mm}$ o.d.) with epoxy glue was used to remove Type 2 SEPs from the GC's injector. Conditioned SEPs were stored individually or together in tubes (150 $\mathrm{mm} \times 18 \mathrm{~mm}$ i.d.) with screw caps or B14 ground-glass stoppers. Loaded SEPs were stored individually in storing tubes (140 mm $\times 2.0 \mathrm{~mm}$ i.d.) with B-5 ground glass stoppers or screw caps with 8-mm Teflon-faced septa, or were sealed off in an oxy-propane burner equipped with a small nozzle.

\section{Sampling and analytical procedures}

Single analyses of standard gas samples were carried out according to the basic procedure described by Burger et al. [5]. A centrally pierced septum was placed in a spare septum cap (cool) of the GC. Septa that have previously been used for conventional liquid injection are perfect for this purpose. The back end of the stalk of the SEP was inserted into the septum from its Teflon-faced side and the septum and cap were moved to a position on the stalk that would put the PDMS sleeve of the installed SEP halfway between the top and bottom of the GC's injector liner. This position was marked on the stalk. The resulting combination of SEP, septum and cap was used as a unit during conditioning, sampling and analysis [5]. To install the SEP in the injector, the carrier gas was turned off and the (hot) septum cap and septum were removed from the injector. Holding the previously described SEP-septum-cap assembly by the SEP's stalk, the SEP was lined up vertically with the orifice of the septum-supporting insert and without delay released to fall sharply into the injector, after which the septum cap was tightened, the carrier gas turned on and the analysis started.

The SEP is left in the injector until the analysis has been completed and the oven has cooled down to the ambient temperature. In the present study it was not necessary to re-adjust the position of the SEP on its stalk after sampling gas mixtures. However, if the glassware and closures described in our previous publication [5] are used to enrich volatiles from the headspace of liquid samples, it might be necessary to have the PDMS sleeve at a higher position in the sample vessel in order to avoid droplets of a rapidly stirred liquid sample being splashed onto the sleeve. Before the SEP is installed in the injector, the septum and cap then have to be re-positioned using a bench mark on a cool part of the GC or another clean surface. In standard SEP practice, simultaneous sampling in multiplicate can be carried out using an appropriate SEP holder. A B-29 drip cone was, for example, converted into a 
stopper by sealing off its upper end in a glass blower's torch. Using standard glass blowing procedures, four or more glass prongs were attached to the lower rim of the drip cone. The stalks of the SEPs were fastened to these prongs using aluminium foil strips. Alternatively, a glass SEP holder with four parallel vertical slits can be used. The slits ensure vertical alignment of the SEPs, which are fastened to the holder with an aluminium foil strip (Supplementary information, Fig. S1).

However, any other non-sorptive holding or clamping device could be used in a similar manner to hold the required number of SEPs during sampling (Fig. S2). Losses of highly volatile analytes from loaded SEPs were restricted by transferring the SEPs to storing tubes as quickly as possible. In the case of the multiplicate sample enrichment mentioned in the previous paragraph, the B-29 cone with the four attached SEPs was placed upside-down and the storing tubes were each dropped open-end-down onto the SEPs (Fig. S3). With the sleeves of the SEPs still in the storing tubes, the back ends of their stalks were extracted from the aluminium foil strips, the tubes were capped and the SEPs were stored at $-10^{\circ} \mathrm{C}$ until analysed. To restrict losses of the volatile analytes from the PDMS sleeve even further, as required in exploratory experiments with highly volatile analytes, the SEPs, while still attached to the drip cone or the SEP holder, were cooled for a few seconds to $c a .-80^{\circ} \mathrm{C}$ in the headspace gas of liquid nitrogen in a $2.5-1$ Dewar before they were introduced into the storing tubes, and stored at $-10{ }^{\circ} \mathrm{C}$ until analysed.

SEPS that had been stored at low temperatures were removed and installed in the injector in the following manner. While holding the tube by its upper part to avoid warming the lower part of the tube and the sleeve, the upper end of the SEP's stalk was partly withdrawn from the tube and fed through the septum and cap (Fig. S4). With the lower part of the SEP still in the cold tube, the position of the septum on the stalk was adjusted using marks previously made on the tube (Fig. S5), after which the SEP was removed from the tube and dropped into the injector. In addition to these standard sampling procedures, some extraordinary measures were taken specifically to locate and then eliminate any possible causes of the unacceptable RSDs obtained in quantitative multiplicate analyses [5]. For example, gas samples were stirred during the sampling period to avoid the formation of thermally based concentration gradients in the large sampling vessels that were used.

A 20-I round-bottom flask with a single B-42 ground glass socket was thermally insulated in a cardboard box with polystyrene foam. The flask was fitted with a B-42 drip cone, which on its upper end had a B-29 socket carrying a B29 glass KPG stirrer (Schott, Mainz, Germany). An overhead stirrer motor (Heidolph, Schwabach, Germany) was used to stir the gas mixture in the flask. The flask and other glassware were not silanised. In order to restrict the loss of volatiles from the SEPs on removal from the gas sample and to standardise any losses that were nevertheless likely to occur during the manipulation of the SEPs in multiplicate analyses, the following procedure was followed in a few experiments. Using a narrow strip of adhesive tape, the upper ends of the stalks of up to 20 SEP30s were attached at precisely measured intervals (e.g. $10 \mathrm{~mm}$ ) in a parallel fashion to the outside surface of the lower part of the B-42 drip cone, with the PDMS sleeves extending below the lower rim of the drip cone.

Storing tubes were attached vertically and in a straight line to a stable support at $10-\mathrm{mm}$ intervals, ready to receive the loaded SEPs. After completion of the enrichment step, the stirrer motor and stirrer were removed from the setup, the B-42 drip cone was then quickly removed from the flask and, if thought necessary, the SEPs were cooled in the headspace gas of liquid nitrogen. The adhesive tape with the SEPs still attached to it was then quickly peeled off the drip cone. Using both hands, the adhesive tape was held straight to align the SEPs with the tubes, the adhesive tape was released and each SEP was allowed to drop into its own storing tube. With a quick downward movement the adhesive tape was peeled simultaneously off the SEPs, the storing tubes were closed with their B-5 stoppers or screw caps, and were stored at $-10^{\circ} \mathrm{C}$. This operation is best performed by two or three people.

These precautions were used only in a few experiments and are not advocated for general SEP analyses. Storing tubes were cleaned by heating them at $500{ }^{\circ} \mathrm{C}$ in a glassware annealing oven, but could also be cleaned by rinsing with residue analysis grade dichloromethane and drying them in a GC oven or any other clean drying oven.

\section{Standard gas mixtures}

Standard gas mixtures containing $n$-alkanes and primary alcohols in concentrations of approximately $5 \mu \mathrm{g} / \mathrm{l}$ were prepared from various mixtures of equal quantities of the alkanes and primary alcohols listed in Table 3. An appropriate volume, e.g. $1 \mu \mathrm{l}$, of a neat mixture of standards was either injected into a 10- or 20-I thermally isolated round-bottom flask, or the mixture was introduced into the flask using a glass or fused silica capillary (0.2 $\mathrm{mm}$ i.d.) that was long enough to reach the bottom of the round-bottom flask. An appropriate volume (graduated length) of the mixture was allowed to enter the capillary (capillary forces) and was then blown out of the capillary onto a tiny piece of filter paper in the bottom of the flask. The capillary was quickly withdrawn from the flask, the flask was closed with the appropriate sampling hardware described above, and then left at $22{ }^{\circ} \mathrm{C}$ for at least $24 \mathrm{~h}$ before the analytes were sampled. 


\section{Determination of carry-over}

In order to determine the extent of carry-over that could be expected with the new SEP30s when a temperature program of $4{ }^{\circ} \mathrm{C} / \mathrm{min}$ is used, a mixture of standards was sampled simultaneously with eight Type 1 SEPs. The loaded SEPs were stored and analysed as described above. The analyses were interrupted and the SEPs removed 1.0, 1.5, 2, 3, 5, 7 and 10 min after the individual analyses had been started. In each of these analyses the SEP was removed from the injector and stored as described above. The injector was closed with the spare septum cap and the current analysis was allowed to run to completion, after which the stored SEP was re-installed in the injector for quantitative analysis of the material that was still undesorbed when the first analysis had been interrupted. The percentage of undesorbed material remaining in the sorptive phase when the SEPs were removed from the injector was calculated from the quantitative results of each set of these analyses.

\section{Results and discussion}

\section{Hardware modifications and SEP handling procedures}

The original SEPs consisted of relatively thick PDMS sleeves on 1.5-mm stainless steel stalks (Fig. 1). Using a thinner PDMS sleeve on a thinner stalk in order to produce a SEP with a stalk that would pass through a standard injector cap and a sleeve that would pass through the septum-supporting injector insert were an obvious way of circumventing changes to the injector hardware for the implementation of the SEP technique. The manufacturing of SEPs was simplified using fused silica tubing $(0.7 \mathrm{~mm}$ o.d.) as stalks instead of the previously employed stainless steel rods (Fig. 1). Increasing the sleeve length from previously $15 \mathrm{~mm}(0.028 \mathrm{~g})$ to $30 \mathrm{~mm}$ compensated for the resulting smaller volume of sorptive phase per unit length of PDMS tubing. Although the diameter of the stalk of the finished product was smaller than that of the needle-guiding hole of the septum cap, the sleeve still did not pass sufficiently rapidly through the standard septum-supporting insert of the injector.

Nevertheless, the new SEPs were easier and cheaper to manufacture than the SEPs of the first generation. However, the most important advantage of using the new SEPs is that highly volatile compounds are eluted as sharp peaks, in contrast to the partly split peaks described in our previous publication [5]. The flowless period that was previously used to circumvent the problem of split peaks thus became redundant, although some tailing of the early eluting compounds was still observed. A typical gas chromatogram of a mixture of the $24 n$-alkanes and primary alcohols listed in Table 3 is depicted in Fig. 2. High-boiling compounds were also desorbed more rapidly from the thinner sleeves, which practically eliminates the risk of carry-over from one analysis to the next. Experiments showed that even octadecane is quantitatively desorbed from the thinner sleeves in less than $10 \mathrm{~min}$ at an injector temperature of $230^{\circ} \mathrm{C}$.

The results of these experiments shown in Table 1 are intended to serve only as an approximate guideline because the method that was used to quantify the carry-over has the drawback that small proportions of the analytes, even of high-boiling analytes such as octadecane, are probably lost from the SEP on its removal from the hot injector. With the new SEP, carry-over could only become a problem when very high temperature programming rates are used. Exploratory experiments should thus be carried out to determine whether this risk exists in applications requiring the utilisation of high temperature programming rates.

\section{Reproducibility}

The moderate cost of SEPs has made multiple simultaneous sampling with a plurality of SEPs an attractive alternative to carrying out consecutive analyses with the same SEP in investigations such as the present one. In our previous paper on the SEP technique [5] the relatively high RSDs that were obtained in such multiplicate analyses were tentatively ascribed to the possibility that the SEPs had different thermal histories. If the standard procedure is followed, SEPs spend extended periods in the injector during analyses and during the time it takes the column to cool down after the completion of analyses. These periods may vary with varying programming rates and the duration of isothermal and conditioning periods. The difference in thermal ageing of the PDMS sleeves could thus possibly have produced PDMS sleeves with different sorptive properties.

In order to evaluate this hypothesis, fifteen SEP30s with sleeves falling within a weight variance of $0.14 \%$ were made. The SEPs were each conditioned for precisely $20 \mathrm{~min}$ and placed in labelled storing tubes. The SEPS thus had identical thermal histories. The SEPs were exposed to a gas sample containing approximately equal proportions of eight alkanes, ranging from pentane to dodecane. Except for the thermal isolation of the flask from which the analytes were sampled, the special precautions mentioned above were not applied. Analyses of the sorbed volatiles produced RSDs similar to those obtained with SEPs of the first generation, which refuted the theory that differences 
in the thermal history of the SEPs could be responsible for high RSDs. This matter was further investigated by conditioning one of the above-mentioned SEPs for $2700 \mathrm{~h}$ at $230{ }^{\circ} \mathrm{C}$ in the injector of a GC with hydrogen as carrier gas. The quantitative results obtained with this SEP were not different from those obtained with the others.

Although the SEP that had been subjected to this long-term conditioning was not periodically removed from the injector and exposed to the atmosphere, and its long-term conditioning could therefore not be equated to normal practice in which the hot PDMS sleeve of a SEP is repeatedly exposed to the atmosphere, it was nevertheless unlikely that the high RSDs could be ascribed to differences in the thermal histories of the SEPs. This result is in line with observations showing that there is no deterioration in the performance of SBSE stir bars after 100 extractions [4]. Contrary to standard analytical practice, which requires replication of the total analytical procedure, analyses in multiplicate were carried out in the present investigation by simultaneous sampling from the same sample vessel.

This was done to rule out the contribution to the high RSDs of parameters other than those having an immediate bearing on the performance of the SEPs. If multiple simultaneous sampling is employed, storing some of the loaded SEPs cannot be circumvented. Experiments were thus carried out to determine whether the quality of quantitative data could be influenced by the conditions under which SEPs are stored. SEPs stored at $22{ }^{\circ} \mathrm{C}$ in B- 5 glass tubes gave low recoveries (Fig. 3), probably because B-5 joints are not absolutely gas tight. As expected, SEPs stored at low temperatures, even in B-5 tubes, gave excellent recoveries. Immediately after completion of the enrichment step, loaded SEPs should thus be placed in the smallest possible storing tubes and, for long-term storing, sealed off using a glass blower's burner, or capped with screw caps and Teflon-faced septa.

Loaded SEPs can be stored without loss of analytes for extended periods at $-10{ }^{\circ} \mathrm{C}$ in gas-tight tubes. It must be kept in mind that the penetration of all analytes into the PDMS sleeve is not an instantaneous process. When analytes are present in an analytical sample in such low concentrations that enrichment has to be carried out for extended periods of several hours or days, the loss of analytes from the loaded SEPs during the few seconds it takes to introduce the loaded SEPs into their storing tubes will not have a major influence on the resulting RSDs. If, on the other hand, the analytes are present in high concentrations and sampling takes only a few minutes, post-enrichment SEP handling, storing of SEPs as quickly as possible and avoiding any irregularities in handling SEPs become critically important. In order to restrict the loss of highly volatile analytes from the PDMS during these few critical moments, the SEPs can therefore also be cooled in the headspace gas of liquid nitrogen for a few seconds immediately after their removal from the gas sample before they are stored at $-10{ }^{\circ} \mathrm{C}$ in capped storage tubes.

Experiments were carried out to study the possible effect of exposing loaded SEPs to the atmosphere on the retention of analytes in the sorptive phase. In order to obtain quantifiable losses exceeding the inherent limits of reproducibility of the SEP technique at the stage at which these experiments were carried out, SEPs were exposed to the atmosphere at $22{ }^{\circ} \mathrm{C}$, as well as to the headspace gas of liquid nitrogen at $-80^{\circ} \mathrm{C}$ for much longer periods than it would normally take to transfer a SEP from the sample vessel to a storing tube or from the storing tube to the GC's injector. The results presented in Fig. 4 prove that variations in the duration of the exposure of SEPs to the atmosphere or to cold nitrogen gas could influence the reproducibility of the technique, especially as far as highly volatile analytes are concerned. An analyst experienced in the SEP technique could probably transfer three or four SEPs from a SEP holding device to storing tubes within about $3 \mathrm{~s}$, using the procedure explained in Section 2, and could close the tubes in another $5 \mathrm{~s}$.

Cooling before storing three or four loaded SEPs thus probably does not make any significant contribution towards lower RSDs, but it could improve RSDs when sampling with ten or more SEPs. It is reiterated that this precaution is only of value in multiple simultaneous SEP sampling of highly volatile analytes. In normal SEP practice it is rarely necessary to go to such extreme lengths. Unsatisfactory RSDs could also be ascribed to random losses of volatile analytes when SEPs are introduced into the injector. In our previous publication on this topic [5], we showed that highly volatile compounds are already largely desorbed by the time the septum cap has been properly tightened. It is therefore possible that varying proportions of volatile analytes could be lost by diffusion from the injector if no particular attention is devoted to the timing of the steps required for the installation of a SEP, closing of the injector and turning on the carrier gas flow. Furthermore, varying quantities of the already desorbed volatiles could be purged from the injector via the split line by pressure surges resulting from manual opening of the carrier gas shutoff valve in a haphazard manner.

This is illustrated by the results given in Table 2. By cooling loaded SEPs immediately on removal from a standard gas mixture and carrying out analyses on the Trace GC with its electronic carrier flow control, marginally lower RSDs were obtained for the lower-boiling analytes. As shown in Table 2, electronic carrier gas flow control also ensured 
high retention time reproducibility. In efforts to reduce the number of manipulations required for the installation of a SEP, experiments with Type 2 SEPs were carried out. The short stalks of these SEPs made it possible to drop them into the injector and to close the injector with the septum cap without having to first insert the back end of the SEP into the septum and septum cap.

The PDMS sleeves of these SEPs were positioned $20 \mathrm{~mm}$ from the lower tip of the stalk, which ensured that the SEPs would come to rest in the injector with their sleeves at least $10 \mathrm{~mm}$ above the tip of the capillary column. After completion of an analysis these SEPs were extracted from the injector with a strong magnet (Fig. S6). Surprisingly, analyses done in this way gave RSDs that were much higher than those obtained in previous analyses. About $75 \%$ of the analyses gave quantitative results that were grouped relatively closely together, whereas the other analyses gave a random scatter of much lower values. Close observation of the behaviour of the SEP as it moves down into the injector revealed that SEPs did not always move into the injector at exactly the same speed. Some SEPs tended to execute a sort of corkscrew motion or vibration, while moving visibly slower into the injector. This was captured on film at relatively slow shutter speed (Fig. S7).

It was impossible to completely straighten the coiled guitar string and it is possible that slightly bent stalks, exacerbated by the lightness of the Type 2 SEPs, could have been responsible for the slower downward movement of some SEPs through the hot septum-supporting insert and the premature desorption of analytes. To eliminate this problem, a guide consisting of two tiny in-line glass funnels was lined up with the septum-supporting insert before introduction of the SEP. However, very little consistent improvement was observed (Table 3). Apparently the mass of the septum cap is an essential prerequisite for the fast downward movement of the SEP and obtaining acceptable RSDs. Although the idea of using Type 2 SEPs has not been given up it has not been pursued further in the present investigation. It was concluded that standardising the sample handling and SEP introduction procedures was probably the best way of improving the reproducibility of the technique.

Earlier experiments have shown that the longer it takes to install the septum cap, the more material is lost from the injector. The results in Table 3 show that satisfactory RSDs can be obtained even when the septum cap installation procedure is intentionally drawn out a few seconds and more material is lost during the extra seconds, provided the procedure is accurately timed and carried out in a strictly controlled and reproducible manner. However, to keep such losses within limits in trace analyses, the injector should nevertheless be closed as quickly as possible, for example by reducing the number of wrist and finger movements required to tighten the septum cap.

One way of achieving this is using a shallower septum cap or an appropriately thicker septum, and by dropping the cap onto the injector in a position in which its thread would immediately interlock with that of the injector (Fig. S8). If an appropriately thick septum is used, the cap can then be tightened within about one quarter of a turn. Using a bayonet type of locking device could be a further alternative solution to this problem.

\section{Sensitivity of the technique}

As the amount of an analyte extracted from a gas sample depends on the volume or mass of the sorptive medium, the approximately $29 \mu \mathrm{l}$ of PDMS on a SEP30 can be expected to extract nearly 60 times the amount extracted by the $0.5 \mu$ l of PDMS on a $100 \mu \mathrm{m}$ SPME fibre under identical equilibrium sampling conditions. In a few practical applications, in which sampling was not necessarily carried out under equilibrium conditions, we have found a SEP30 to be approximately 40 times as sensitive as a $100 \mu \mathrm{m}$ PDMS fibre. As far as the enrichment step is concerned, the SEP technique and HSSE are practically identical and the results obtained by Tienpont et al. with the latter technique [7] could be used as practical guidelines for the prediction of the sensitivity of the SEP technique, keeping in mind that these authors used $51.5 \mu$ l of PDMS. These techniques are so sensitive that capillary columns can easily be overloaded with analytes that are present in high concentrations in analytical samples.

In such cases accurate timing of essentially quite short enrichment periods is important in quantitative work. The temperature profiles of the injectors of the GCs used in this investigation are quite flat, i.e. the injector wall has practically the same temperature over its entire length, with the exception of the few millimeters at the very top and bottom of the injector. This allows the use of longer PDMS sleeves than the standard $30 \mathrm{~mm}$ of SEP30 s. In order to establish the maximum length of PDMS sleeve that could be used, a series of SEPs were made having sleeve lengths that ranged from $10 \mathrm{~mm}$ to $80 \mathrm{~mm}$. The SEPs were conditioned and simultaneously introduced into a gas sample containing the alkanes and primary alcohols used in some of the previous experiments. The loaded SEPS were simultaneously removed from the gas sample, stored in the storing tubes described above, and analysed in random order. 
Within the reproducibility limits of the technique, linear relationships were found between the mass of the PDMS and the quantities of the analytes sorbed in it. Thus SEP80s were about 2.6 times more sensitive than SEP30s. Nevertheless, it is not advisable to compare quantitative results obtained with a SEP30 with those obtained using a SEP50, for example. The positioning of the sleeve in the injector could be critical in work with long-sleeved SEPS and until more experience has been gained with long-sleeved SEPs, sleeves longer than $60 \mathrm{~mm}$ should preferably be used only for qualitative work. A better understanding of the parameters that could impact on the reproducibility of the SEP technique opens up the possibility of increasing the sensitivity still further by reverting to the use of thicker PDMS sleeves in analyses of analytes with boiling points higher than that of octane.

In other applications the problematic splitting of the peaks of highly volatile analytes when thick sleeves are used, could be circumvented using capillary columns with higher stationary film thicknesses, provided the possibility of carry-over of high-boiling compounds is kept in mind.

\section{General guidelines}

The steps that could be taken to ensure quantitatively reproducible results can be summarised as follows:

Sampling should be carried out from thermostatted or thermally insulated sample vessels. Depending on the boiling points of analytes, sampling should preferably not be carried out at temperatures much higher than room temperature because it is more difficult to control the reproducibility of the loss of volatiles from a hot PDMS sleeve than from a cooler sleeve during transfer to a storing tube or to the GC's injector. Sampling of headspace volatiles from aqueous media should be carried out at a temperature low enough to avoid the formation of condensed water droplets on the PDMS sleeve because the sorption of especially apolar analytes is hampered by the water.

Loaded SEPs must be transferred as quickly as possible from the sampling vessel to the GC or GC-MS injector or, in the case of multiple simultaneous sampling, to holding tubes. Loaded SEPS should be stored at low temperatures, for example at $-10^{\circ} \mathrm{C}$. Storing SEPs in an ice box during field work is better than not cooling the SEPs at all. It is possible that a small plug of silanised glass fibres placed about $15 \mathrm{~mm}$ from the lower end of the injector liner could compensate for small variations in the position a SEP in the injector.

The septum and septum cap must be accurately positioned on the SEP's stalk, using a benchmark in a cool place, conveniently on or near the GC. This could also be done with the sleeve still in the cold storing tube and while holding the tube by its upper part to avoid warming the lower part of the tube and the sleeve by hand. The position of the septum and septum cap on the SEP's stalk can be adjusted while the SEP is still in a cold storing tube using marks on the storing tube.

As described in Section 2, the carrier gas is shut off and the (hot) septum cap and septum are removed, the still cool storing tube is held vertically next to the injector with the arrow on the cap lined up with a mark on the GC as explained above, the SEP with the septum-septum cap assembly is taken out of the tube, held vertically in line with the orifice of the septum supporting insert and is then dropped (not inserted) into the injector, after which the septum cap is tightened. The carrier gas is turned on without delay (without employing a flowless period), after which the temperature programme and data acquisition are started.

It is reiterated that some of the extraordinary precautions discussed above, such as stirring gas samples and cooling SEPs in the headspace gas of liquid nitrogen, are not advocated for general use in SEP analyses.

\section{Concluding remarks}

The SEP technique was developed primarily for high-capacity sorptive sample enrichment in qualitative applications. In analyses of the territorial marking fluid of the Bengal tiger, Panthera tigris, compounds ranging from hexane to squalene were identified using the SEP technique [8] and in work on the neonatal recognition pheromone of sheep, Ovis aries, compounds up to Cholest-5-en-3 3 -ol were determined using SEP50s and sample enrichment at $70{ }^{\circ} \mathrm{C}$ for an hour (Burger et al., unpublished). The technique could also be a cost-effective alternative to existing sorptive enrichment techniques in quantitative applications, specifically in applications requiring multiple simultaneous sampling.

The sampling of air pollution in or around chemical plants and the comparison of air pollution profiles at many different points in a city are examples that spring to mind. We have demonstrated that acceptable RSDs can be obtained in quantitative applications by rigorously controlling the handling of loaded SEPs and their introduction into the GC or GC-MS injector, i.e. by adopting a reproducible sample handling and introduction 'rhythm'. The 
results shown in the last column of Table 3 were obtained by an operator with a few weeks' experience in SEP analysis. Therefore, using this technique, it should not be difficult to routinely achieve RSDs that are comparable to those obtained with other sorptive enrichment techniques. The possibility of an ongoing investigation of the use of Type 2 SEPs has been mentioned above.

Finding acceptable solutions for the problems mentioned above in connection with the use of Type 2 SEPs could also simplify the implementation of the SEP technology on current Agilent GC models in which the carrier gas is introduced into the injector via the septum cap. The insertion of the stalk of a Type 1 SEP into the septum and the introduction of the PDMS sleeve into the injector of these GCs are so awkward that, at present, large scale losses of the enriched volatiles cannot be avoided.

The development of the SEP technique was to a large extent motivated by the niche that exists in research on pheromones and other semiochemicals, fragrances and flavours for a simple, flexible and affordable analytical technique for projects requiring limited numbers of analyses on a seasonal or intermittent basis. Automation of the SEP technique, which would probably result in the development of expensive instrumentation, has therefore not yet been seriously considered. If a project calls for an automated high-throughput technique, SBSE technique would probably the method of choice. 


\section{Tables}

Table 1.

Carry-over of analytes with respect to the time the SEP spends in the injector at $230{ }^{\circ} \mathrm{C}^{\mathrm{a}}$.

\begin{tabular}{|c|c|c|c|c|c|c|c|}
\hline \multirow{3}{*}{ Analytes } & \multicolumn{7}{|c|}{ Carry-over per analyte (\%) } \\
\hline & \multicolumn{7}{|c|}{ Desorption time in the injector $(\mathrm{min})$} \\
\hline & 1.0 & 1.5 & 2.0 & 3.0 & 5.0 & 7.0 & 10 \\
\hline Nonane & nd & nd & nd & nd & nd & nd & nd \\
\hline 1-Hexanol & 0.26 & nd & nd & nd & nd & nd & nd \\
\hline 1-Octanol & 0.35 & 0.15 & nd & nd & nd & nd & nd \\
\hline Undecane & 0.23 & 0.09 & nd & nd & nd & nd & nd \\
\hline Undecanol & 0.69 & 0.31 & 0.02 & 0.03 & 0.02 & nd & nd \\
\hline Tetradecane & 0.88 & 0.27 & 0.07 & 0.05 & nd & nd & nd \\
\hline Heptacecane & 3.46 & 1.34 & 0.09 & 0.06 & 0.03 & nd & nd \\
\hline Heptacecane & 5.31 & 2.39 & 0.21 & 0.11 & 0.1 & nd & nd \\
\hline
\end{tabular}

nd, not detected.

a Results were obtained by multiplicate sampling and GC analysis of a mixture of selected alcohols and alkanes at concentrations of $c a .5 \mu \mathrm{g} / \mathrm{l}$, timed desorption in the injector and analysis, followed by desorption and analysis of the analytes remaining in the PDMS sleeve of the SEP30 after the first desorption cycle.

b Time the SEP spent in the injector at $230^{\circ} \mathrm{C}$.

Table 2.

Comparison of RSDs obtained in SEP analyses carried out under various experimental conditions ${ }^{\text {a }}$.

\begin{tabular}{|c|c|c|c|c|c|c|}
\hline \multirow{3}{*}{ Analytes } & \multicolumn{6}{|c|}{ RSDs } \\
\hline & \multicolumn{2}{|c|}{ Manual carrier gas shut-off ${ }^{b}$} & \multicolumn{4}{|c|}{ Electronic carrier gas controlc } \\
\hline & $\begin{array}{c}\text { Inaccurate } \\
\text { controld,e } \\
(n=7)\end{array}$ & $\begin{array}{c}\text { Accurate } \\
\text { controle,f }(n=3)\end{array}$ & $\begin{array}{c}\text { Normal SEP } \\
\text { handlingg }(n=5)\end{array}$ & $\begin{array}{l}\text { Cooled SEPsh } \\
\quad(n=5)\end{array}$ & Rt (min) & $\begin{array}{l}\text { Rt deviation } \\
\text { (min) }\end{array}$ \\
\hline Pentane & 24.49 & 16.52 & 20.36 & 7.52 & 1.230 & 0.005 \\
\hline Hexane & 9.88 & 8.74 & 12.30 & 10.08 & 2.238 & 0.005 \\
\hline Heptane & 19.20 & 8.86 & 7.62 & 7.84 & 3.640 & 0.006 \\
\hline Octane & 37.87 & 4.59 & 2.20 & 5.97 & 5.409 & 0.008 \\
\hline Nonane & 8.05 & 2.82 & 2.19 & 4.82 & 7.301 & 0007 \\
\hline Decane & 11.58 & 1.33 & 3.65 & 4.19 & 9.166 & 0.007 \\
\hline Undecane & 17.79 & 0.65 & 4.81 & 4.94 & 10.949 & 0.006 \\
\hline Dodecane & 24.33 & 0.15 & 4.67 & 5.44 & 12.630 & 0.007 \\
\hline Tridecane & & & 4.29 & 5.33 & 14.210 & 0.007 \\
\hline Tetradecane & & & 4.47 & 5.11 & 15.695 & 0.007 \\
\hline Pentadecane & & & 4.67 & 4.74 & 17.097 & 0.007 \\
\hline Hexadecane & & & 4.75 & 4.16 & 18.426 & 0.005 \\
\hline Heptadecane & & & 4.79 & 3.47 & 19.680 & 0.008 \\
\hline Octadecane & & & 5.19 & 2.00 & 20.877 & 0.006 \\
\hline
\end{tabular}

a Results obtained by simultaneous sampling with Type $1 \mathrm{SEP} 30 \mathrm{~s}$ at $22^{\circ} \mathrm{C}$ from a gas mixture containing alkanes in concentrations of approximately $5 \mu \mathrm{g} / \mathrm{l}$. GC analyses were carried out using a fused silica column: $33 \mathrm{~m} \times 0.32 \mathrm{~mm}$, 1.2 2 m PS-255 (DB-1 equivalent).

b Carlo Erba HRGC, programmed from $35^{\circ} \mathrm{C}$ to $280^{\circ} \mathrm{C}$ at $4{ }^{\circ} \mathrm{C} / \mathrm{min}$, split flow $19 \mathrm{ml} / \mathrm{min}$, septum purge $6 \mathrm{ml} / \mathrm{min}$. c Thermo Trace GC, programmed from $45^{\circ} \mathrm{C}$ to $265^{\circ} \mathrm{C}$ at $10{ }^{\circ} \mathrm{C} / \mathrm{min}, 6 \mathrm{~s}$ splitless, followed by a split flow of $10 \mathrm{ml} / \mathrm{min}$.

d Inaccurately timed flowless period and carrier gas flow turned on rapidly. 
e Experiment carried out with samples containing only $n$-alkanes from pentane to dodecane.

f Precise timing of the duration of the flowless period, and careful opening of carrier gas shut-off valve.

g Normal sample handling without rapid cooling of SEPs in an atmosphere of liquid nitrogen after sorptive enrichment.

h Rapid cooling of SEPS after sorptive enrichment.

Table 3.

Selection of results obtained in SEP analyses under various instrumental conditions ${ }^{\mathrm{a}}$.

\begin{tabular}{|c|c|c|c|c|c|}
\hline \multirow{3}{*}{ Analytes $^{\mathbf{b}}$} & \multicolumn{5}{|c|}{ Relative standard deviation (RSD) } \\
\hline & \multicolumn{2}{|c|}{ Without glass wool in liner SEP30 Type $2^{c}$} & \multicolumn{3}{|c|}{ With glass wool in liner SEP30 Type $1^{d}$} \\
\hline & \multirow[t]{2}{*}{ Without guide $(n=14)$} & \multirow[t]{2}{*}{ With guide $(n=4)$} & \multicolumn{2}{|c|}{ Cap closure delay } & \multirow{2}{*}{\begin{tabular}{|c|}
$\begin{array}{c}\text { Optimised SEP } \\
\text { introduction }\end{array}(n=12)$ \\
\end{tabular}} \\
\hline & & & $3 s(n=8)$ & $6 \mathrm{~s}(n=8)$ & \\
\hline Hexane & 12.02 & 13.45 & 2.63 & 3.64 & 6.59 \\
\hline Heptane & 12.78 & 2.46 & 0.24 & 6.55 & 6.13 \\
\hline 1-Butanol & 25.74 & 15.20 & 4.70 & 7.52 & 12.42 \\
\hline Octane & 8.11 & 2.00 & 0.20 & 5.43 & 4.25 \\
\hline 1-Pentanol & 24.27 & 10.65 & 1.93 & 3.07 & 5.72 \\
\hline Nonane & 6.13 & 2.51 & 1.02 & 3.58 & 3.80 \\
\hline 1-Hexanol & 8.52 & 10.98 & 1.54 & 1.32 & 4.74 \\
\hline Decane & 4.64 & 2.40 & 1.62 & 1.84 & 3.08 \\
\hline 1-Heptanol & 7.76 & 6.58 & 1.40 & 1.23 & 4.39 \\
\hline Undecane & 5.01 & 7.71 & 1.61 & 1.64 & 2.64 \\
\hline 1-Octanol & 6.80 & 9.20 & 1.22 & 1.16 & 3.49 \\
\hline 1-Nonanol & 6.07 & 10.03 & 4.70 & 7.52 & 2.28 \\
\hline Dodecane & 5.92 & 11.09 & 1.45 & 1.78 & 2.19 \\
\hline 1-Decanol & 6.69 & 5.86 & 0.83 & 1.93 & 1.56 \\
\hline Tridecane & 6.93 & 11.81 & 1.48 & 2.30 & 1.62 \\
\hline 1-Undecanol & 6.65 & 0.99 & 0.08 & 2.39 & 1.10 \\
\hline Tetradecane & 7.51 & 10.37 & 0.78 & 2.54 & 1.56 \\
\hline 1-Dodecanol & 10.19 & 3.23 & 0.75 & 2.40 & 1.17 \\
\hline Pentadecane & 6.19 & 6.65 & 0.41 & 3.36 & 1.84 \\
\hline 1-Tridecanol & 26.48 & 1.33 & 2.34 & 3.82 & 1.49 \\
\hline Hexadecane & 11.08 & 1.78 & 2.45 & 4.35 & 2.96 \\
\hline 1-Tetradecanol & 27.22 & 10.38 & 5.58 & 6.04 & 2.89 \\
\hline Heptadecane & 13.99 & 2.52 & 4.51 & 5.33 & 3.78 \\
\hline Octadecane & 11.88 & 17.07 & 6.12 & 6.84 & 5.17 \\
\hline
\end{tabular}

a Results obtained by simultaneous sampling with Type $1 \mathrm{SEP} 30 \mathrm{~s}$ at $22^{\circ} \mathrm{C}$ from a gas mixture containing $n$-alkanes and primary alcohols in concentrations of approximately $5 \mu \mathrm{g} / \mathrm{l}$. GC analyses were carried out on a Carlo Erba HRGC, programmed from $35^{\circ} \mathrm{C}$ to $280^{\circ} \mathrm{C}$ at $4{ }^{\circ} \mathrm{C} / \mathrm{min}$; split flow $19 \mathrm{ml} / \mathrm{min}$; septum purge $6 \mathrm{ml} / \mathrm{min}$ using a fused silica column: $33 \mathrm{~m} \times 0.32 \mathrm{~mm}, 1.2 \mu \mathrm{m}$ PS-255 (DB-1 equivalent).

b In order of increasing boiling points.

c SEP30 Type 2 with short stainless steel stalk and with the PDMS sleeve $20 \mathrm{~mm}$ from the tip of the stalk.

d SEP30 Type 1 with fused silica stalk with normal length of $130 \mathrm{~mm}$.

e Time elapsed between SEP introduction and tightening of septum cap.

f With implementation of the basic guidelines mentioned in the text. 


\section{Figures}

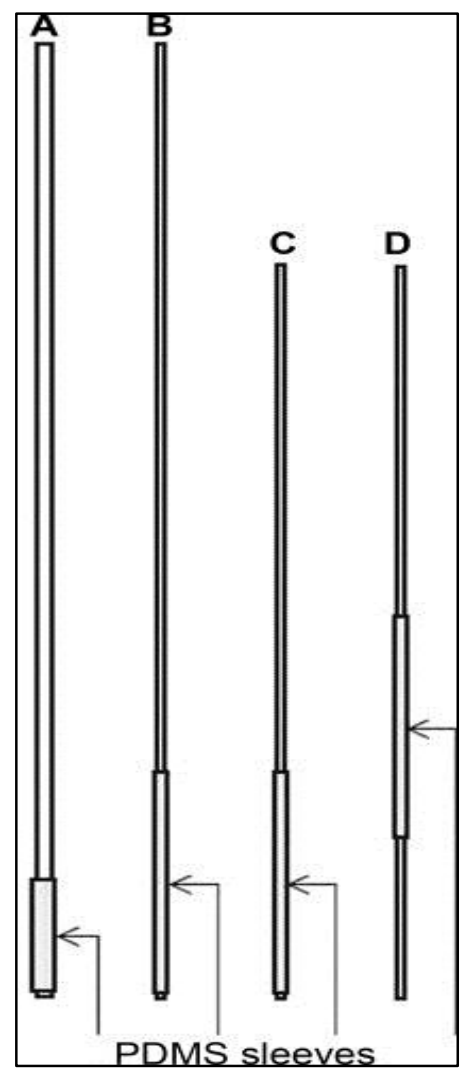

Fig. 1. Schematic illustration of various SEP designs. (A) SEP of the first generation $(130 \mathrm{~mm} \times 1.5 \mathrm{~mm}$ stainless steel stalk with $15 \mathrm{~mm} \times 1.00 \mathrm{~mm}$ i.d. $\times 1.75 \mathrm{~mm}$ o.d. PDMS sleeve); (B) Type 1 SEP of the second generation (130 mm $\times 0.7 \mathrm{~mm}$ polyimide-coated fused silica stalk with $30 \mathrm{~mm} \times 0.64 \mathrm{~mm}$ i.d. $\times 1.19 \mathrm{~mm}$ o.d. PDMS sleeve); (C) Type 2 SEP of the second generation $(100 \mathrm{~mm} \times 0.66 \mathrm{~mm}$ paramagnetic stainless steel stalk with $30 \mathrm{~mm} \times 0.64 \mathrm{~mm}$ i.d. $\times 1.19 \mathrm{~mm}$ o.d. PDMS sleeve); and (D) Type 2 SEP of the second generation similar to $\mathrm{C}$ but with the sleeve positioned $20 \mathrm{~mm}$ from the tip of the stalk.

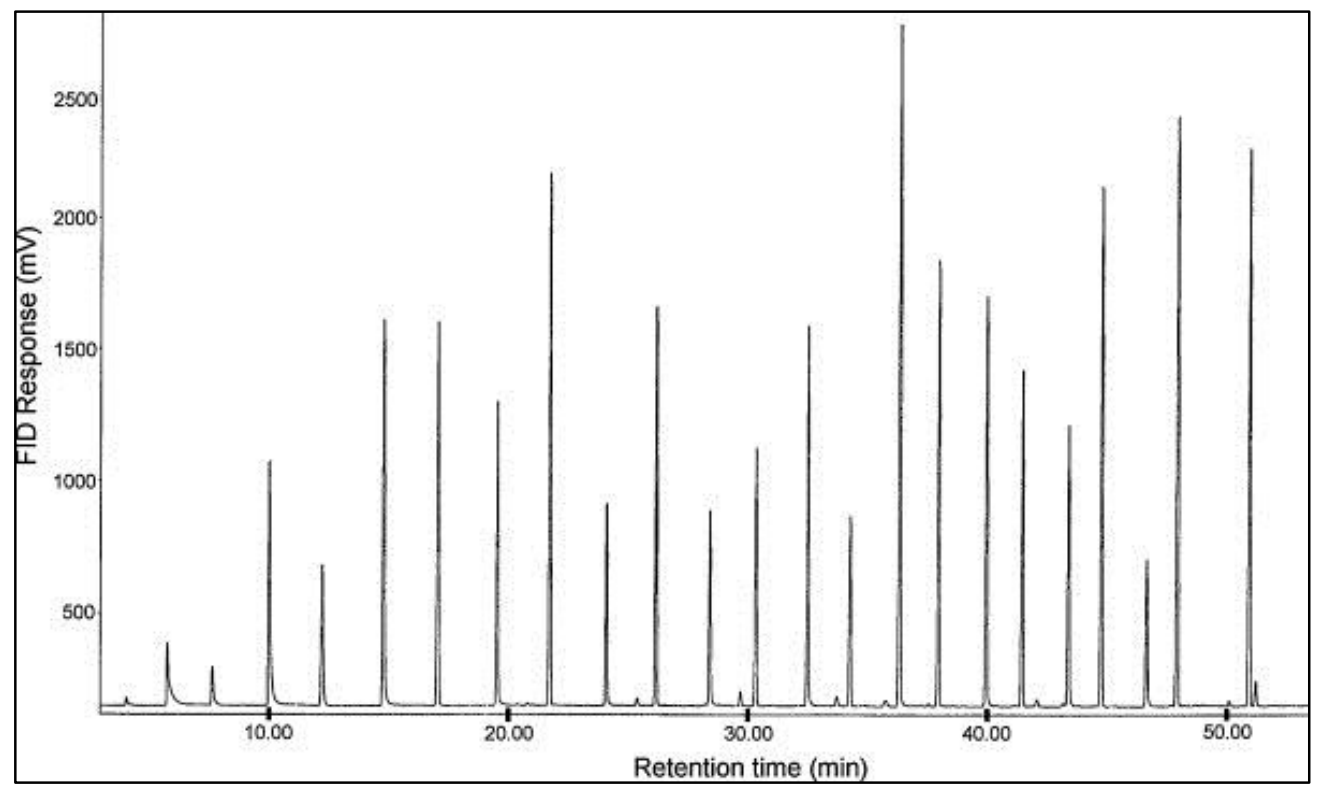

Fig. 2. Gas chromatogram of the $n$-alkanes and primary alcohols listed in Table 3 . Fused silica column: $30 \mathrm{~m} \times 0.25 \mathrm{~mm}$ i.d. coated with $1.2 \mu \mathrm{m}$ of PS-255 (DB-1 equivalent), made with a 5-m retention gap; columns programmed from $30^{\circ} \mathrm{C}$ to $280^{\circ} \mathrm{C}$ at $4{ }^{\circ} \mathrm{C} / \mathrm{min}$; carrier gas hydrogen under constant pressure and at a linear flow velocity of $50 \mathrm{~cm} / \mathrm{s}$ at $40^{\circ} \mathrm{C}$. 


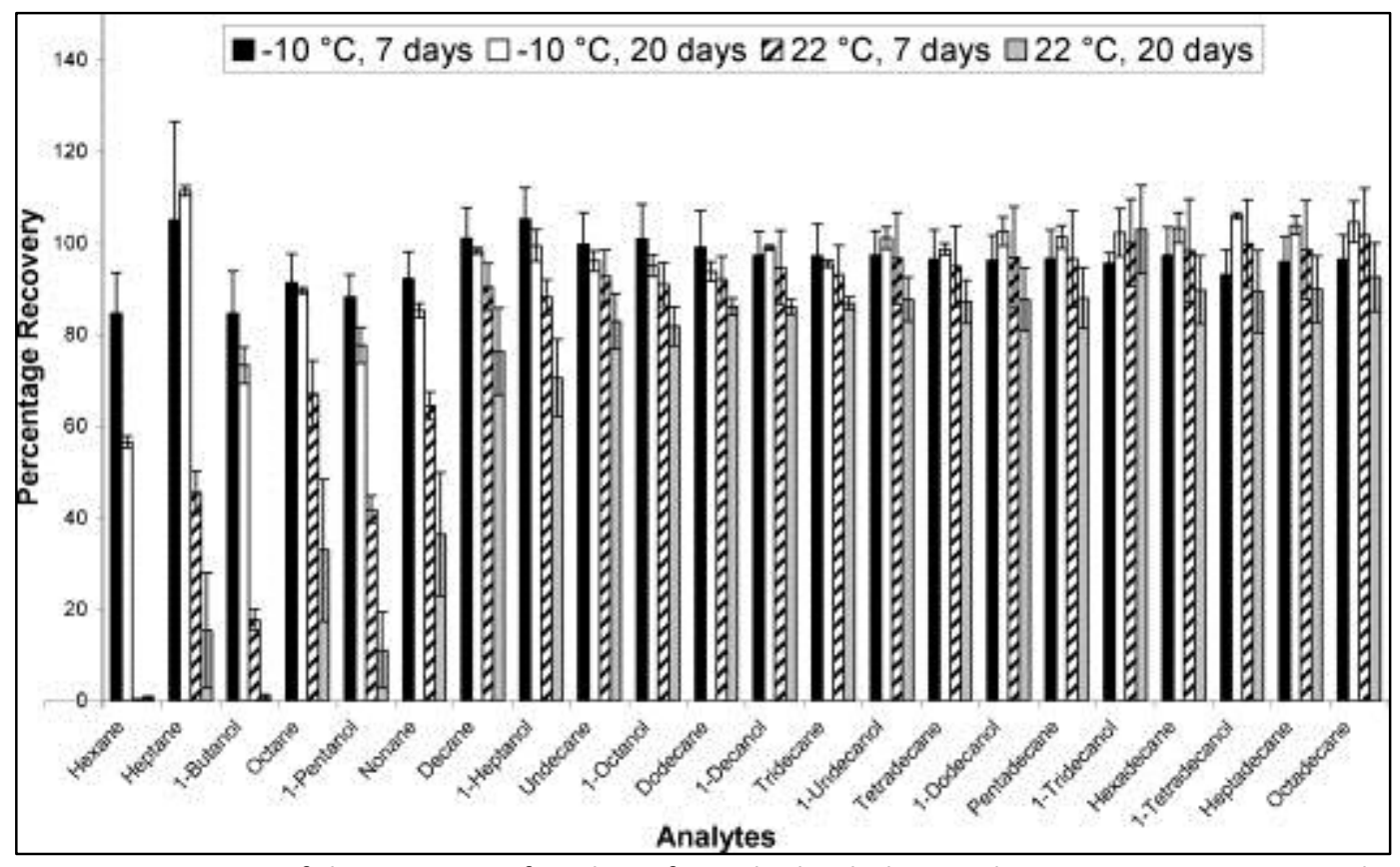

Fig. 3. Comparison of the recovery of analytes from the loaded second generation SEP30s stored in storing tubes capped with B-5 stoppers at $22^{\circ} \mathrm{C}$ in the laboratory atmosphere and at $-10{ }^{\circ} \mathrm{C}(n=3)$. Values are given relative to recoveries from SEP30s stored in tubes capped with screw caps and Teflon-faced septa at $-10{ }^{\circ} \mathrm{C}$, which were taken as taken as $100 \%$.

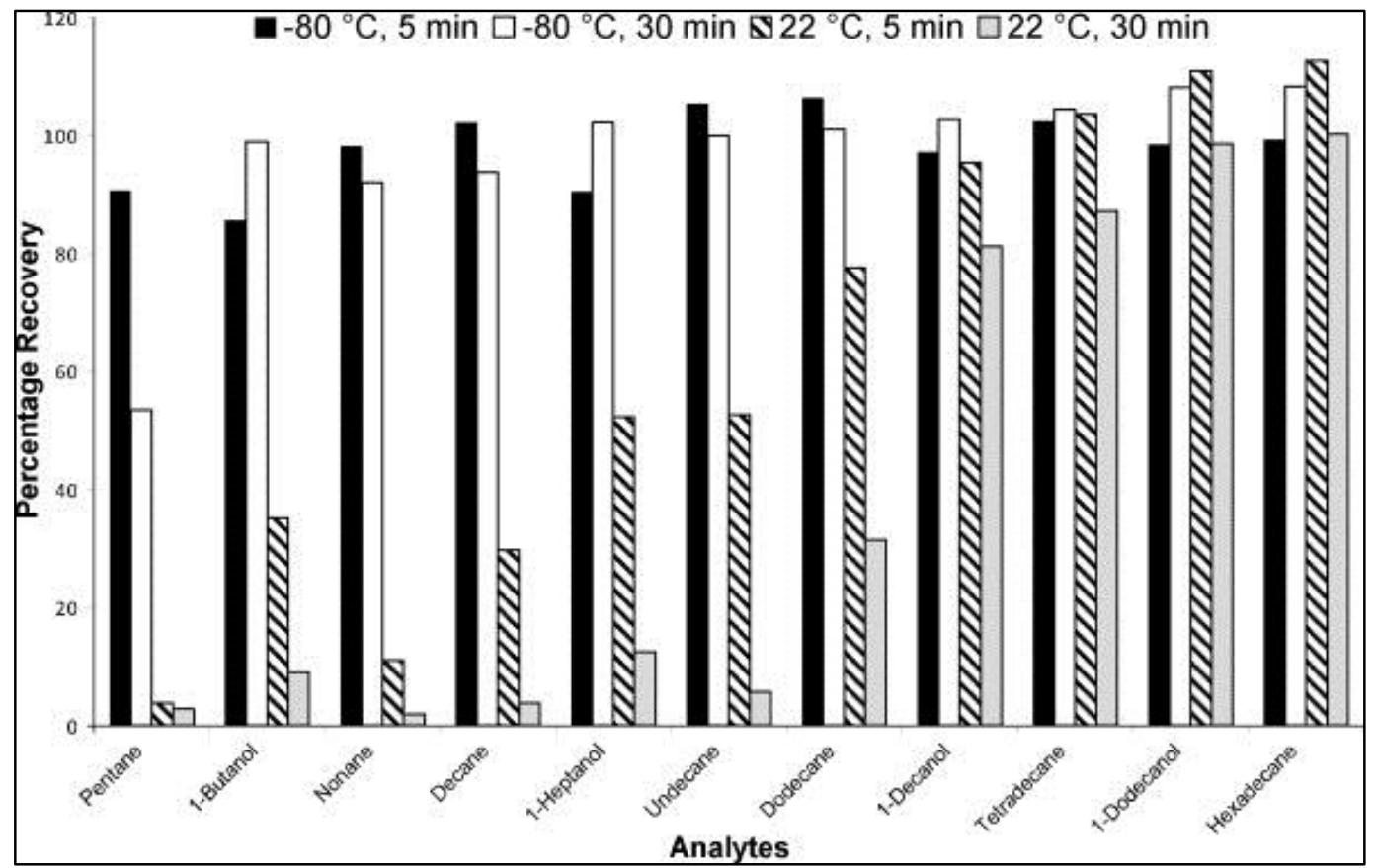

Fig. 4. Comparison of the recovery of analytes from the loaded second generation SEP30s exposed to laboratory air and to the headspace gas of liquid nitrogen at $-80^{\circ} \mathrm{C}$ for periods of 5 and $30 \mathrm{~min}$. 


\section{References}

1. B.V. Burger, Z.M. Munro J. Chromatogr., 370 (1986), p. 449

2. C.L. Arthur, J. Pawliszyn Anal. Chem., 62 (1990), p. 2147

3. E. Baltussen, H.-G. Janssen, P. Sandra, C.A. Cramers J. High Resolut. Chromatogr., 20 (1997), p. 385

4. E. Baltussen, P. Sandra, F. David, C. Cramers J. Microcolumn Sep., 11 (1999), p. 737

5. B.V. Burger, B. Marx, M. le Roux, W.J.G. Burger J. Chromatogr. A, 1121 (2006), p. 259

6. C. Bicchi, C. Cordero, C. Iori, P. Rubiolo, P. Sandra J. High Resolut. Chromatogr., 23 (2000), p. 539

7. B. Tienpont, F. David, C. Bicchi, P. Sandra J. Microcolumn Sep., 12 (2000), p. 577

8. B.V. Burger, M.Z. Viviers, J.P.I. Bekker, M. le Roux, N. Fish, W.B. Fourie, G. Weibchen J. Chem. Ecol., 34 (2008), p. 659 